\title{
Expert Consensus on Nurses' Human Caring for COVID-19 Patients in Different Sites*
}

\author{
Feng-jian ZHANG ${ }^{1 \dagger}$, De-ying HU ${ }^{1 \dagger}$, Yi-lan LIU ${ }^{1 \#}$, Hong $\mathrm{LI}^{2}$, Xiao-ping ZHU ${ }^{3}$, Shao-shan PAN ${ }^{4 \#}$ \\ ${ }^{1}$ Union Hospital, Tongji Medical College, Huazhong University of Science and Technology, Wuhan 430030, China \\ ${ }^{2}$ Fujian Provincial Hospital, Fujian 350001, China \\ ${ }^{3}$ Zhongnan Hospital, Wuhan University, Wuhan 430071, China \\ ${ }^{4}$ General Hospital of Southern Theatre Command of China People's Liberation Army, Guangzhou 510010, China
}

(C) Huazhong University of Science and Technology 2020

\begin{abstract}
Summary: This study aims to develop the expert consensus on nurse's human caring for Corona Virus Disease 2019 (COVID-19) patients in different sites, and thus provide a guideline on providing whole process and systematic caring for COVID-19 patients. Based on the frontline experiences of human caring for COVID-19 patients and the review of literature, the initial draft of consensus was made and finalized after online meeting and revisions. The experts reached consensus on the following parts: terms and definitions, principles of human caring for COVID-19 patients, and human caring measures for COVID-19 patients in different sites. The expert consensus is practical, concise, and reasonable for guiding the nurses providing human caring for COVID-19 patients, as well as other similar infectious diseases.
\end{abstract}

Key words: human caring; nursing; expert consensus; COVID-19

The current novel Corona Virus Disease 2019 (COVID-19) outbreak, which began in December 2019, presents a significant challenge for the entire world ${ }^{[1]}$. With a high infection rate, rapid spread, and uncertain clinical course duration, the COVID-19 can lead infected patients into critical conditions, and even death $^{[2]}$. The unexpected infection of COVID-19 not only threatens the patient's health and life, but also seriously affects the patient's psychological state ${ }^{[3-5]}$, such as the fear and irritability caused by the uncertainty and the unknown outcome of the disease, the loneliness and helplessness resulting from the prohibiting of visit by family members and friends during hospitalization and isolation, the anxiety and depression in knowing about the infection of their relatives and friends. The patients with COVID-19 may go through several stages in different sites, such as fever clinic visiting, mobile cabin hospital, designated hospital treatment,

Feng-jian ZHANG, E-mail: zfj1234567789@gmail.com; De-ying Hu, E-mail: hudeying2006@126.com

${ }^{\dagger}$ The authors contributed equally to this work.

\#Corresponding authors, Yi-lanLIU,E-mail:yilan12020@163. com; Shao-shan PAN, E-mail: panssh@163.com

*This study was supported by Project of Technological Innovation of Hubei Province (Soft Science Research) (No. 2018ADC078) and Project of Independent Innovation of Double-First Class Construction (Art Interdisciplinary) (No. 5001530064) centralized medical isolation observation, home isolation, even rehabilitation clinic visiting ${ }^{[6-11]}$. Regardless of the sites and the stages, patients are continuously exposed to the difficulties and pressure, and prone to negative emotions and psychological problems, which strongly call for personalized human caring ${ }^{[12,13]}$.

Currently, many nursing experts have explored the human caring measures for COVID-19 patients in different sites, but the standardized and whole-process human caring measures for COVID-19 patients are not available. Therefore, the experts were invited to develop a consensus on delivering human caring in different sites, thus to provide guideline for the human caring to COVID-19 patients as well as other similar infectious diseases during the whole process of treatment and recovery.

\section{METHODS OF CONSTRUCTING THE EXPERT CONSENSUS AND THE SCOPE OF THE CONS- ENSUS}

\subsection{Methods of Constructing the Expert Consensus}

Nursing administrators from Hubei, Fujian, Beijing, Sichuan, Shanghai, Guangdong, conducted a systemic search and thereafter collected the related literature from several electronic databases: PsychINFO, CINAHL, PubMed, Web of Science and 
CNKI (Chinese). The keywords include nursing, human caring, caring, etc. The draft of the consensus was made after reviewing and summarizing the literature, as well as collecting the frontline experiences and measures of human caring for COVID-19 patients. Two rounds of interdisciplinary expert meetings by invitation were organized in April 2020 to revise the draft and finally reached the consensus. Thirty-two experts from multiple medical institutions participated.

\subsection{The Scope of the Consensus}

The consensus can be applied in different sites (fever clinic, hospital isolated ward, mobile cabin hospital, isolation observation center, rehabilitation clinic) where COVID-19 patients get medical treatment. Moreover, it can also be used as guideline for patients with other similar infectious diseases in different sites.

\section{CONTENTS OF THE EXPERT CONSENSUS}

\subsection{Terms And Definitions}

COVID-19 refers to the disease caused by the novel coronavirus began in 2019.

Human caring, attention, and understanding of human nature, which satisfies human needs, protects human dignity and interests, and embodies human value from the perspective of human needs and desires.

Fever clinic, a specific medical area in the outpatient department of a hospital during the prevention and control of acute infectious diseases according to the instructions of the superior. It is particularly established for the screening and diagnosis of suspected or infected patients, and provides a specific medical area for observation and treatment of patients in need.

Hospital isolated ward is the medical department for the treatment of severe and critical patients with COVID-19.

Isolated unit in mobile cabin hospital is the temporarily built and renovated medical institution for the temporary treatment of the COVID-19 patients with taking the Chinese People's Liberation Army (PLA)'s field mobile medical system as a model.

Centralized medical isolation observation centers are the specialized places for medical isolation and observation of COVID-19 patients after discharge and before returning home.

Rehabilitation clinic is a special established institution that provides services for patients with COVID-19 during the discharged recovery stage, such as consultations, follow-ups, and physical and mental rehabilitation.

\subsection{Principles of Human Caring for COVID-19 Patients in Different Sites}

(1) Nurses should be highly aware of the severity and complexity of COVID-19, as well as the difficulty and suffering of patients, to protect patients' lives and health and make them feel warmly supported.

(2) Nurses should be responsible and kind-hearted to patients, treat patients as if they are the family members, or even assume the roles of their family members if necessary.

(3) Integrate human caring into the whole process of treatment and recovery, while meeting patient's individualized human caring needs.

(4) Deliver human caring with professional and creative measures $^{[14]}$.

(5) Treat nurses themselves as the most important resource, and comprehensively utilize the available resources to provide human caring ${ }^{[15]}$.

(6) Highlight the mobilization of patients' selfcare and motivate their potential capacity to facilitate self-healing, and encourage the support and human caring between patients ${ }^{[16]}$.

(7) Pay attention to patients about the infection of their family members, and provide prompt psychological support if the infection or death occurs.

(8) Nursing staff should conduct standard procedure of self-care and self-protection in order to ensure both physical and mental health, and report any negative feelings and emotions, as well as symptoms, therefore, to avoid the occurrence of relevant accidental incident.

(9) Write or stick the name and role noticeably on the outer protective suit to make it easy for patients to identify and call.

\subsection{Human Caring in Different Sites}

\subsubsection{Human Caring for Patients in Fever Clinic}

(1) Receive patients forwardly and warmly with polite manner and moderate voice, and introduce the name and role to patients; (2) Provide masks for patients and their companions, and teach them the right way to put on the mask; (3) The triage nurse should arrange patients visiting orderly, and timely register the basic information, then measure and record their vital signs. Prioritize the appointment of critical patients, recognize the abnormal symptoms of patients in time, and assist in treatment if necessary ${ }^{[17]}$; (4) The triage nurse should actively provide guidance and help to patients, assist patients with critical conditions or mobility problems in registration, physical examination, picking up medicine; (5) Keep the environment clean, make the guidance signs clear, decorate with proper posters, and disinfect regularly; (6) Provide comfortable seats for waiting patients. Ensure one room, one physician and one patient during consultation to prevent crossinfection and protect patients' privacy; (7) Identify the negative emotions of patients, care for the depressed patients, comfort the emotional patients, and provide psychological support. Take on the role of their family members for the patients lacking family support because of their families suffering from COVID-19. Accompany the patients to make them feel safe 
and warm; (8) Visit patients frequently during the treatment, care for the patients forwardly and assess the health status actively, and take measures to relieve the pain and discomfort. Remind the patients of their safety to avoid falls, and tell the patients to call for help from the nurses if needed; (9) Arrange the patients in observation reasonably according to their conditions, and provide a single room when possible. Patients with common fever, suspected infection, and confirmed COVID-19 infection, should be classified and managed in different ways by following guidelines; (10) Assess the basic needs of patients, and provide warm food and warm water according to their needs. Report the unsolved problems to head nurse or seek help from other departments; (11) Set up a green channel for critical patients. Assist in transferring the patient to other areas or hospitals according to the conditions; (12) Record and hand over the patients' special caring needs or measures to the next shift;

\subsubsection{Human Caring for Patients in Hospital} Isolation Ward (1) Receive patients forwardly and warmly with polite manner and moderate voice, and introduce the name and duty to the patients; (2) The duty nurse should daily communicate with the patients appropriately at the bedside. Assess the needs of patients proactively and fulfill their reasonable needs. The unsolved problems by nurses should be reported to the head nurse, doctor or other related people to seek help; (3) Identify the patient's physical discomfort through monitoring, questioning, and observing. Apply professional knowledge and skills to relieve patients' suffering and facilitate their comfort, even organize relevant specialist nursing consultation; (4) Communicate proactively with the patients and listen to them in daily work, and evaluate the psychological status with PHQ-4 if necessary to figure out the abnormal conditions. Timely comfort, accompany and encourage the patients with anxiety and fear, even act as a temporary role of patient's family member, and the psychologist can be invited to help to deal with the particular problems. Encourage and facilitate the caring and communication between the patients, and assist the patient in communicating with their families and friends; (5) Deliver nursing operations normatively, correctly and timely, and inform and communicate with patients during the operation, even if the patients are unconscious. Close the door or cover the patients body during the operation to protect the patients privacy; (6) Provide necessary knowledge of disease and health to the patients. Provide and instruct the patients to put on masks. Teach the recovering patients to start breathing training to promote the lung function; (7) Assist the patients in daily life and medical treatment, and prepare daily necessities for them. The items provided by their relatives and friends should be delivered to the patients timely. When the patients get out of bed, the nurses should pay close attention or give support to avoid accidents such as falling. (8) Pay attention to the nutrition of patients' diets, prepare delicious and warm meals, and try the best to provide personalized meals according to patients' needs. Prepare fruits, milk and other foods appropriately. Provide microwave ovens in the ward for heating foods at any time. Encourage and assist patients without swallowing disorders to eat by themselves. Provide enteral and parenteral nutrition for patients with eating problems. Take away the left food and plates as soon as patients finish eating; (9) Keep the wards clean and warm, and ventilate and disinfect regularly; (10) Assist in or implement bed bath and other hygiene care for the patients to keep clean and comfortable; (11) Encourage the patients in the recovery rehabilitation and activities involvement, and express the appreciation for the patient cooperation; (12) Record the special caring needs or measures of patients on the whiteboard and handover between each shift; (13) Provide wake-up service for coma patients four times a day, and play audio materials with patients' favorite music, the blessing, and encouraging words from relatives, colleagues, and friends; (14) Provide detailed guidance on medication, isolation, condition report, and follow-up for discharged patients. Provide channels for consultation such as telephone or message platforms; (15) Provide support to the patients in their terminal stage. Express respect to the dead while delivering death care. Inform their family members about the unfortunate news and related information with appropriate manner ${ }^{[18]}$.

\subsubsection{Human Caring for Patients in Mobile Cabin}

Hospital (1) Receive patients forwardly and warmly with polite manner and moderate voice, and introduce the name and duty to the patients; (2) Guide the patients to the cabin and assist with taking the luggage if needed; (3) Introduce the environment and staff information to the patients, especially the functional area like doctors' office, nurse station, toilet, bathroom, to facilitate their adaptation to the environment; (4) Create a friendly living environment, maintaining cleanness, disinfecting regularly, and ventilating efficiently. Decorate the isolated unit with positive and inspirational posters and slogans to encourage patients to fight with the disease, and use proper equipment to protect the patients privacy; (5) Enquire about the patients needs on living and medical service, provide necessary supplies for their daily use, and improve the service and facilities for living environment. Ensure patients get living belongings from family members as soon as possible. Report to the head nurse or other managers if there are any unsolved problems; (6) Greet patients at the bedside on shift change and handover the patients' special caring demands and measures between nurses; (7) Implement treatment measures correctly and timely, and protect 
patient's privacy during operating procedure; (8) Visit the patient regularly, report to the doctor if the patient gets worse, and assist with the patient's transition when needed; (9) Organize diverse recreational activities to enrich the patients' daily life in the cabin. Encourage the patients to participate in activities or volunteer work, thus to make them feel valuable and confident with recognition and encouragement; (10) Evaluate the mental status of the patient, comfort the patient who feels anxiety or down, and assist them in communicating with their families and friends in different ways according to their needs, ask the psychologist for help if necessary; (11) Assist the patient to sort out items and provide personalized discharge guidance upon discharge. Thereafter, lead the patient to the doorway with appreciation and best wishes, and hand over with community staff.

\subsubsection{Human Caring for Patients in Centralized} Medical Isolated Observation Centers (1) Treat the patient warmly and politely with cordial greeting, and assist in carrying personal belongings to the room; (2) Introduce the environment and living facilities to the patient, as well as the precautions and work procedures to facilitate adaptation; (3) Keep the room clean, hygienic, and warm, disinfect the room regularly; (4) Establish convenient and multi-party communication channels for nurses and patients to make it easy for the patient to report the living needs, disease change, and treatment needs at any time ${ }^{[19]}$; (5) Assess the patient's physical conditions and medical service needs daily and provide health education and suitable treatment; (6) Assist the patient in seeking medical treatment outside the cabin hospital; (7) Provide convenient services like delivering items, assisting in receiving medicines, purchasing items, and guiding medication usage. Assist the isolated person with life difficulties or selfcare disabilities and assist them in eating, washing, and doing activities. Ensure the accompanying guardian and safety of the children; (8) Guide the isolated person to start appropriate rehabilitation activities such as lung function rehabilitation exercises; (9) Teach the methods of isolation and disinfection at home to the isolated person, and explain the knowledge of disease prevention to improve the awareness and ability of protection; (10) Assess the psychological status of the patient, encourage peer support and family engagement in the psychological support for the patient.

\subsubsection{Human Caring for Patients with Home}

Quarantine (1) Conduct regular follow-up to
assess the discharged patient's recovery and needs with politeness. Leave a message or call again in $2 \mathrm{~h}$ for the lost-call; (2) Inquire about the health status, medication use, check-up, diet, and rehabilitation of the patients, and provide corresponding guidance and help; (3) Assess the patient's isolation conditions and protection measures, and remind the patient if necessary; (4) Provide home care for the patient when needed. Remind the patient to seek medical help if any patient's condition changed dramatically, and arrange the ambulance to transfer the patient while necessary; (5) Listen to the patient, answer the patient's questions patiently, and provide help within the ability during the follow-up. Seek help and support from the relevant specialist for special difficulties; (6) Relieve the patient's bad mood, and show empathy to the patient's suffering during the follow-up. Invite the psychologist or relevant specialist to intervene for severe psychological problems; (7) Assist patients in arranging further consultation and other matters; (8) Record the patient's special problems or conditions for other healthcare workers to pay attention to in future follow-up; (9) Provide necessary assistance for patients to return to their work;

\subsubsection{Human Caring for Patients in Rehabilitation}

Clinic (1) Receive patient forwardly and warmly with polite manner and moderate voice, and introduce the name and role to the patients; (2) Keep the clinic area spacious, and decorate the area with clear signs, warm colors, green plants, and positive energy posters; (3) Design a quick physical examination process, guide the recovered person to finish the examination at different times. Provide personalized examination program for the recovered people like elderly people, infants, postpartum women and those with basic diseases; (4) Provide a comfortable environment with the necessary facilities. Ensure that the waiting area is equipped with specialized seats for the recovered person to rest, and other supplies like hand sanitizer, towel paper, disposable cups, medical masks, wheelchairs should be provided. The heating equipment should be equipped and set to an appropriate temperature; (5) Provide nutritional, safe, and warm food catering for the patients at a specific time; (6) Take care of the patient when the patient is rehabilitating, and stop the rehabilitation in time if the patient feels uncomfortable. Additionally, handle emergencies happened to the patient timely; (7) Assess the health needs of patients, launch various health education programs, and provide health guidance, such as dietary guidance, sleeping adjustment, self-monitoring after taking medication, caring for COVID-19 related symptoms, person and home environment disinfection methods, to improve patients' self-care ability ${ }^{[20]}$; (8) Teach the recovered patient to master the six-character breathing tactics and traditional Chinese medicine exercises, such as Baduanjin and Taijiquan, to improve the lung function and immunity function; (9) Pay attention to the psychological and emotional state of the patients. The nurse can use relevant scales to evaluate and find abnormalities during this process, and intervene timely. Ask the psychologist to intervene for special psychological problems; (10) Make connections 
actively with the community, hospitals, and other institutions to provide extended care for the patient. Conduct remote rehabilitation and health guidance by phone, WeChat, and videoes to provide support for patient treatment and recovery according to the needs of the patient.

\section{Acknowledgements}

The authors would like to show respects to all nursing staff who providing human caring to patient with COVID-19 in pandemic. Thanks for Ms Suoling Zhou from Baylor College of Medicine, USA, for her great support on polishing the language.

\section{Conflict of Interest Statement}

The authors have no conflict of interest.

\section{Appendix}

Expert Consensus Panel on Nurses' Human Caring for COVID-19 Patients in Different Sites (in alphabetical order of surname)

Zhong-xiang CAI (Renmin Hospital, Wuhan University); A-dan FU (Central Hospital of Wuhan, Tongji Medical College, Huazhong University of Science and Technology); Qiu-ying HAN (Zhongshan Hospital, Xiamen University);

Jiao HE (Union Hospital, Tongji Medical College, Huazhong University of Science and Technology);

De-ying HU (Union Hospital, Tongji Medical College, Huazhong University of Science and Technology);

Jie HU (Union Hospital, Tongji Medical College, Huazhong University of Science and Technology);

Liu HU (Wuhan Asia Heart Hospital);

Yan JIANG (West China Hospital, Sichuan University);

Yan JIN (Union Hospital, Tongji Medical College, Huazhong University of Science and Technology);

Hong LI (Fujian Provincial Hospital);

Ling LI (Union Hospital, Tongji Medical College, Huazhong University of Science and Technology);

Xiao-ning LI (Wuhan Tangjiadun Community Health Center);

Zhi-ying LI (The First Affiliated Hospital, Sun Yat-sen University);

Wei-hua LIU (Wuhan Jinyintan Hospital);

Xiao-juan LIU (Beijing Chaoyang Hospital, Capital Medical University);

Yi-lan LIU (Union Hospital, Tongji Medical College, Huazhong University of Science and Technology);

Gen-di LU (Shuguang Hospital, Shanghai University of Traditional Chinese Medicine);

Cai-hong LU (Union Hospital, Tongji Medical College, Huazhong University of Science and Technology);

Shao-shan PAN (General Hospital of Southern Theatre Command of China People's Liberation Army);

Qing-lian SHI (Dongguan People's Hospital);

Chang-xiu WAN (Hubei Provincial Hospital of TCM, Hubei University of Chinese Medicine);

Wei-xian WANG (Union Hospital, Tongji Medical College, Huazhong University of Science and Technology);

Li-fen WU (Union Hospital, Tongji Medical College, Huazhong University of Science and Technology);

Han XIONG (Union Hospital, Tongji Medical College,
Huazhong University of Science and Technology);

Rong XU (Tongji Hospital, Tongji Medical College, Huazhong University of Science and Technology);

Xi XU (General Hospital of Southern Theatre Command of China People's Liberation Army);

Yu-lan XU (Union Hospital, Tongji Medical College, Huazhong University of Science and Technology);

Juan XU (Union Hospital, Tongji Medical College, Huazhong University of Science and Technology);

Xia YANG (Union Hospital, Tongji Medical College, Huazhong University of Science and Technology);

Wen-jing YU (Union Hospital, Tongji Medical College, Huazhong University of Science and Technology);

Feng-jian ZHANG (Union Hospital, Tongji Medical College, Huazhong University of Science and Technology);

Xiao-ping ZHU (Zhongnan Hospital, Wuhan University)

\section{REFERENCES}

1 Sohrabi C, Alsafi Z, O'Neill N, et al. World Health Organization Declares Global Emergency: A Review of the 2019 Novel Coronavirus (COVID-19). Int J Surg, 2020,76:71-76

2 Deng S, Peng H. Characteristics of and Public Health Responses to the Coronavirus Disease 2019 Outbreak in China. J Clin Med, 2020,9(2):575

3 Cheung T, Fong T, Bressington D. COVID-19 under the SARS Cloud: Mental Health Nursing during the Pandemic in Hong Kong. J Psychiatr Ment Health Nurs, 2020. doi: 10.1111/jpm.12639. Online ahead of print

4 Overton J, Denton K, Frumovitz M, et al. Coronavirus (COVID-19): Patient experience-Administrative Services on the Frontline During Crisis. Head Neck, 2020. DOI: 10.1002/hed.26259

5 Fan PEM, Fazila A, Lim SH, et al. Needs and concerns of patients in isolation care units - learnings from COVID-19: A reflection. World J Clin Cases, 2020, 8(10):1763-1766

6 National Health Commission of the People's Republic of China. Nursing Standards for Patient with COVID-19 in Severe or Critical Conditions [EB/OL]. (2020-03-01) [2020-04-25]. http://www.nhc.gov.cn/yzygj/s7653p/20 2003/8235a35f35574ea79cdb7c261b1e666e.shtml.

7 State Council of the People's Republic of China. Guideline for Prevention and Control of COVID-19 in Medical Institutions [EB/OL]. (2020-01-22) [2020-04-25]. http://www.gov.cn/zhengce/zhengceku/2020-01/23/ content_5471857.htm.

8 National Health Commission of the People's Republic of China. Diagnosis and Treatment Program for Patient with COVID-19 (7th Edition) [EB/OL]. (2020-03-04) [2020-04-25]. http://www.nhc.gov.cn/yzygj/s7653p/20 2003/46c9294a7dfe4cef80dc7f5912eb1989.shtml.

9 National Health Commission of the People's Republic of China. Rehabilitation Program for Discharged Patients with COVID-19 [EB/OL]. (2020-03-05) [2020-04-25]. http://www.nhc.gov.cn/yzygj/s7652ms/202003/377f5fa a9b754debaf3eab7c4de1a9e4.shtml.

10 National Health Commission of the People's Republic of China. Investigation Report of COVID-19 between China and WHO [R/OL]. (2020-02-29) [2020-04-25]. http://www.nhc.gov.cn/jkj/s3578/202002/87fd92510d0 94e4b9bad597608f5cc2c.shtml. 
11 National Health Commission of the People's Republic of China. Strength the Action of Follow-up of Patient with COVID-19 after Discharge [EB/OL]. (2020-0219) [2020-04-25]. http://www.nhc.gov.cn/yzygj/s7653p /202002/0572eef930d5441c96181c44a1 fca878.shtml.

12 Liu YL, Hu DY, Yang C. Theory and Practice of Human Caring in Nursing. Beijing: Peking University Medical Press, 2017.

13 Liu Y, Wu X, Timmins F. Strengthening actions for caring as a core component of nursing in the People's Republic of China. J Nurs Manag, 2019,27(8):15771579

14 Koithan MS, Kreitzer MJ, Watson J. Linking the Unitary Paradigm to Policy through a Synthesis of Caring Science and Integrative Nursing. Nurs Sci Q, 2017, 30(3):262-268

15 Turkel MC, Watson J, Giovannoni J. Caring Science or
Science of Caring. Nurs Sci Q, 2017,31(1):66-71

16 Pitts PJ. Our Most Powerful Weapon to Fight COVID-19: Patient Involvement. Patient, 2020,13(3):255

17 Guan C, Liu Y, Huang H, et al. Outpatient Experience of Human Caring Scale (OEHC-Scale): Improvement by Delphi Method. Curr Med Sci, 2018,38(2):360-371

18 Costantini M, Sleeman KE, Peruselli C, et al. Response and role of palliative care during the COVID-19 pandemic: A national telephone survey of hospices in Italy. Palliat Med, 2020:777080750

19 Wosik J, Fudim M, Cameron B, et al. Telehealth transformation: COVID-19 and the rise of virtual care. J Am Med Inform Assoc, 2020,27(6):957-962

20 Brugliera L, Spina A, Castellazzi P, et al. Rehabilitation of COVID-19 Patients. J Rehabil Med, 2020,52(4):1-3 (Received May 15, 2020; revised July 2, 2020) 